\title{
INFINITE DEFICIENCY IN FRÉCHET MANIFOLDS
}

\author{
BY \\ T. A. CHAPMAN
}

\begin{abstract}
Denote the countable infinite product of lines by $s$, let $X$ be a separable metric manifold modeled on $s$, and let $K$ be a closed subset of $X$ having Property $Z$ in $X$, i.e. for each nonnull, homotopically trivial, open subset $U$ of $X$, it is true that $U \backslash K$ is nonnull and homotopically trivial. We prove that there is a homeomorphism $h$ of $X$ onto $X \times s$ such that $h(K)$ projects onto a single point in each of infinitely many different coordinate directions in $s$. Using this we prove that there is an embedding of $X$ as an open subset of $s$ such that $K$ is carried onto a closed subset of $s$ having Property $Z$ in $s$. We also establish stronger versions of these results.
\end{abstract}

1. Introduction. Let $s$ denote the countable infinite product of open intervals and let $I^{\infty}$ denote the Hilbert cube, i.e. the countable infinite product of closed intervals. A Fréchet manifold (or F-manifold) is defined to be a separable metric space having an open cover by sets each homeomorphic to $s$.

Let $\alpha$ be a set of positive integers and for the remainder of the paper let $T$ denote $s$ or $I^{\infty}$. A subset $K$ of $T$ is said to be deficient with respect to $\alpha$ in $T$ provided that for each of the coordinate intervals which is indexed by an element of $\alpha, K$ projects onto a single interior point of the interval. If $\alpha$ is infinite, then we say that $K$ has infinite deficiency in $T$. More generally let $X$ be a space and let $\alpha$ be as given above. A subset $K$ of $X \times T$ is said to be deficient with respect to $\alpha$ in $X \times T$ provided that $\pi_{T}(K)$ is deficient with respect to $\alpha$ in $T$, where $\pi_{T}$ is the projection of $X \times T$ onto $T$. We say that $K$ has infinite deficiency in $X \times T$ provided that $\pi_{T}(K)$ has infinite deficiency in $T$. We remark that if $X$ is any $F$-manifold, then in [4] it is proved that $X, X \times s$, and $X \times I^{\infty}$ are all homeomorphic.

A closed set $K$ in a space $X$ is said to have Property $Z$ in $X$ if, for each nonempty, homotopically trivial, open set $U$ in $X$, it is true that $U \backslash K$ is nonempty and homotopically trivial. In [2] it is proved that a closed subset $K$ of $T$ has Property $Z$ in $T$ if and only if there exists a homeomorphism $h$ of $T$ onto itself such that $h(K)$ has infinite deficiency in $T$.

If $X$ is any $F$-manifold and $K$ is a closed subset of $X \times T$ having infinite deficiency in $X \times T$, then it is easy to show that $K$ has Property $Z$ in $X \times T$. This follows from the apparatus used in the proof of Theorem 9.1 of [2], which says that infinite deficiency in $T$ implies Property $Z$ in $T$. The question of Property $Z$ implying

Presented to the Society, November 22, 1969; received by the editors July 18, 1969.

AMS Subject Classifications. Primary 5420, 5425.

Key Words and Phrases. The Hilbert cube, Fréchet manifolds, Property $Z$, infinite deficiency.

Copyright (C) 1970, American Mathematical Society 
infinite deficiency in $X \times s$ was raised at the conference on infinite-dimensional topology held at Cornell University, January 5-7, 1969, and it appears as Problem 11 in the report of that meeting, Problems in the topology of infinite-dimensional manifolds. In this paper we solve Problem 11 by proving the following theorem.

THEOREM 1. Let $X$ be any F-manifold and let $\left\{K_{i}\right\}_{i=1}^{\infty}$ be a collection of closed subsets of $X \times T$, with each $K_{i}$ having Property $Z$ in $X \times T$. Then there is a homeomorphism $h$ of $X \times T$ onto itself and a collection $\left\{\alpha_{i}\right\}_{i=1}^{\infty}$, where the $\alpha_{i}$ 's are pairwisedisjoint, infinite sets of positive integers, such that for all $i>0, h\left(K_{i}\right)$ is deficient with respect to $\alpha_{i}$ in $X \times T$.

As an easy consequence of Theorem 1 and the preceding comments we obtain the following characterization of Property $Z$ in $F$-manifolds.

COROLlaRY. Let $X$ be any $F$-manifold and let $K$ be a closed subset of $X$. A necessary and sufficient condition for $K$ to have Property $Z$ in $X$ is that there exists a homeomorphism of $X$ onto $X \times T$ carrying $K$ onto a set having infinite deficiency in $X \times T$.

In [8] David W. Henderson proved that any $F$-manifold can be embedded as an open subset of $s$. In Problem 10 of Problems in the topology of infinite-dimensional manifolds the following question was raised: If, for each $i>0, K_{i}$ is a closed subset of an $F$-manifold $X$ and each $K_{i}$ has Property $Z$ in $X$, is there an open embedding $h: X \rightarrow s$ such that, for each $i>0, h\left(K_{i}\right)$ is closed in $s$ ? In our next theorem we use Henderson's open embedding theorem, modify it with our Theorem 1, and obtain a solution to Problem 10.

THEOREM 2. Let $X$ be any F-manifold and let $\left\{K_{i}\right\}_{i=1}^{\infty}$ be a collection of closed subsets of $X$, with each $K_{i}$ having Property $Z$ in $X$. Regard $s=s_{1} \times s_{2}$, where $s_{1}$ and $s_{2}$ are copies of s, and let $f: X \rightarrow s_{1}$ be any open embedding. Then there exists an embedding $h: X \rightarrow s$ such that $h(X)=f(X) \times s_{2}$ and $h\left(K_{i}\right)$ is a closed subset of $s$ having infinite deficiency in $s$, for all $i>0$.

We note that if $X$ is any $F$-manifold not homeomorphic to $s$, then there is no open embedding $h: X \rightarrow s$ such that $h(X)$ is closed in $s$. Thus the assumption of Property $Z$ in Theorem 2 cannot be omitted.

The author wishes to thank R. D. Anderson for pointing out that Theorem 2 follows from Theorem 1 and for helpful comments on an earlier version of this paper.

2. Preliminaries. We will regard the Hilbert cube $I^{\infty}$ as a canonical compactification of $s$ in which $I^{\infty}=\prod_{i=1}^{\infty} I_{i}$ and $s=\prod_{i=1}^{\infty} I_{i}^{0}$, where for each $i>0$ we have $I_{i}=[-1,1]$ and $I_{i}^{0}=(-1,1)$. The metric we use for $I^{\infty}$ and $s$ is given by

$$
d\left(\left(x_{i}\right),\left(y_{i}\right)\right)=\left(\sum_{i=1}^{\infty} 2^{-i} \cdot\left(x_{i}-y_{i}\right)^{2}\right)^{1 / 2},
$$


where $\left(x_{i}\right),\left(y_{i}\right) \in I^{\infty}$. Whenever no confusion arises we will use $d$ to denote the metric of any space under consideration.

Let $N$ denote the set of positive integers and for each $i \in N$ let $\tau_{i}$ be the projection of $I^{\infty}$ onto $I_{i}$. If $\alpha \subset N$ define $I^{\alpha}=\prod_{i \in \alpha} I_{i}, s^{\alpha}=\prod_{i \in \alpha} I_{i}^{0}$, and let $\tau_{\alpha}$ be the projection of $I^{\infty}$ onto $I^{\alpha}$. For each $i>0$ let $W_{i}^{+}=\tau_{i}^{-1}(1), W_{i}^{-}=\tau_{i}^{-1}(-1)$, and $W_{i}=W_{i}^{+} \cup W_{i}^{-}$. We call $W_{i}^{+}$and $W_{i}^{-}$the endslices of $I^{\infty}$ in the $i$-direction.

A subset of $I^{\infty}$ of the form $\prod_{i=1}^{\infty} J_{i}$ is called a closed basic set in $I^{\infty}$ provided that $J_{i}$ is a closed subinterval of $I_{i}$, for each $i$, and $J_{i}=I_{i}$ for all but finitely many $i$. An open basic set in $s$ is the intersection of a closed basic set in $I^{\infty}$ with $s$.

A homeomorphism $h$ of $I^{\infty}$ onto itself is said to be a $\beta^{*}$-homeomorphism provided that $h(s)=s$. The pseudo-boundary of $I^{\infty}$ is $B\left(I^{\infty}\right)=I^{\infty} \mid s$ and the pseudo-interior of $I^{\infty}$ is $s$.

If $\left\{f_{i}\right\}_{i=1}^{\infty}$ is a sequence of homeomorphisms of a space $X$ onto itself for which the sequence $\left\{f_{i} \circ f_{i-1} \circ \cdots \circ f_{1}\right\}_{i=1}^{\infty}$ converges pointwise to a homeomorphism $f$ of $X$ onto itself, then we call $f$ the infinite left product of $\left\{f_{i}\right\}_{i=1}^{\infty}$ and write $f=L \prod_{i=1}^{\infty} f_{i}$.

We list below three convergence procedures that we will need to insure the existence of an infinite left product of homeomorphisms. The first of these is Lemma 2.1 of [2] and the second is Theorem 2 of [5]. The third is an easy consequence of the apparatus used in [5] to establish the second.

Convergence Procedure A. For each homeomorphism $g$ of a compact metric space $X$ onto itself and each $\varepsilon>0$ let

$$
\eta(g, \varepsilon)=\text { g.l.b. }\{d(g(x), g(y)) \mid d(x, y) \geqq \varepsilon\} .
$$

If $\left\{f_{i}\right\}_{i=1}^{\infty}$ is a sequence of homeomorphisms of $X$ onto itself such that

$$
d\left(f_{i}, \mathrm{id}\right)<\min \left(\left(3^{-i}\right),\left(3^{-i}\right) \cdot \eta\left(f_{i-1}{ }^{\circ} \cdots{ }^{\circ} f_{1}, 2^{-i}\right)\right),
$$

for all $i>1$, then $f=L \prod_{i=1}^{\infty} f_{i}$ exists.

Convergence Procedure B. Let $\mathscr{U}$ be a countable star-finite open cover of any space X. (By star-finite cover we mean a cover such that the closure of each member of the cover intersects the closures of only finitely many other members of the cover.) There exists an ordering $\left\{U_{i}\right\}_{i=1}^{\infty}$ of the elements of $\mathscr{U}$ such that for any sequence $\left\{f_{i}\right\}_{i=1}^{\infty}$ of homeomorphisms of $X$ onto itself, where $f_{i}$ is the identity on $X \backslash U_{i}$ for all $i>0, f=L \prod_{i=1}^{\infty} f_{i}$ exists. Moreover, we can assign a positive integer $n_{i}$ to each $U_{i}$, independent of the choice of $\left\{f_{i}\right\}_{i=1}^{\infty}$, such that $n_{i} \leqq n_{i+1}$ and

for all $i>0$.

$$
f\left(U_{i}\right)=\left(f_{n_{i}} \circ f_{n_{i}-1} \circ \cdots \circ f_{1}\right)\left(U_{i}\right),
$$

Convergence Procedure B'. Let $\mathscr{U}$ be a countable star-finite open cover of any space $X$ and let $\left\{U_{i}\right\}_{i=1}^{\infty},\left\{n_{i}\right\}_{i=1}^{\infty}$ be as in Convergence Procedure B. If $Y$ is any space and $\left\{f_{i}\right\}_{i=1}^{\infty}$ is any sequence of homeomorphisms of $X \times Y$ onto itself such that $f_{i}$ is 
the identity on $\left(X \backslash U_{i}\right) \times Y$, for all $i>0$, then $f=L \prod_{i=1}^{\infty} f_{i}$ exists. Moreover we have

for all $i>0$.

$$
f\left(U_{i} \times Y\right)=\left(f_{n_{i}} \circ \cdots \circ f_{1}\right)\left(U_{i} \times Y\right),
$$

3. Two technical lemmas. We will need a result on extensions of homeomorphisms in $I^{\infty}$, where the given homeomorphism and its extension are required to lie in the same neighborhood of the identity.

Lemma 1. Let $K_{1}, K_{2}$ be compact subsets of $I^{\infty}$ having Property $Z$ in $I^{\infty}$ and let $\varepsilon>0$ be given. If $h: K_{1} \rightarrow K_{2}$ is an onto homeomorphism such that $h\left(K_{1} \cap s\right)=K_{2} \cap s$ and $d(h, \mathrm{id})<\varepsilon$, then $h$ can be extended to a $\beta^{*}$-homeomorphism $H$ such that $d(H, \mathrm{id})<\varepsilon$.

A homeomorphism extension theorem for $I^{\infty}$ was first established by Anderson in [2], and modified with an " $\varepsilon$-condition" in [6] by Barit and in [7] by Bessaga and Pelczyński. If the " $\varepsilon$-condition" is omitted from the statement of our Lemma 1 , then we obtain a statement which is equivalent to a homeomorphism extension theorem of Torunczyk, which is given as Theorem 6 of [9]. If the extension theorem of Barit or that of Bessaga and Pełczyński (loc. cit.) is used, then our Lemma 1 follows routinely from the proof of Torunczyk's extension theorem.

LEMMA 2. Let $\left\{F_{i}\right\}_{i=1}^{\infty}$ be a collection of relatively closed subsets of $s$, with each $F_{i}$ having Property $Z$ in $s$, let $W$ be a finite union of endslices of $I^{\infty}$, and let $\left\{\alpha_{i}\right\}_{i=1}^{\infty}$ be a collection of pairwise-disjoint, infinite sets of positive integers. Then there is $a$ $\beta^{*}$-homeomorphism $h$ and an infinite, increasing sequence $\left\{n_{i}\right\}_{i=1}^{\infty}$ of positive integers such that $h$ is the identity on $W$ and $h\left(\bigcup_{i=1}^{\infty} F_{i}\right)=\bigcup_{i=1}^{\infty} K_{i}$, where each $K_{i}$ is a relatively closed subset of $s$ having deficiency with respect to $\alpha_{n_{i}}$.

Proof. We will construct $h$ as an infinite left product of homeomorphisms $\left\{h_{n}\right\}_{n=1}^{\infty}$. Convergence will be assured by Convergence Procedure A by noting that whenever $h_{1}, \ldots, h_{n}$ have been constructed, $h_{n+1}$ can be constructed arbitrarily close to the identity. We will only explicitly construct $h_{1}$ and $h_{2}$, as the construction of $h_{2}$ will essentially constitute the inductive step.

Let $\beta_{1}=\left\{i_{1}, i_{2}, \ldots\right\}$ be an infinite, increasing sequence of positive integers such that $N \backslash \beta_{1}$ is infinite. We will construct a $\beta^{*}$-homeomorphism $h_{1}$ such that $h_{1}\left(F_{1}\right)$ $=\bigcup_{n=1}^{\infty} A_{n}$, where each $A_{n}$ is a relatively closed subset of $s$ having deficiency with respect to $\alpha_{i_{n}}$, and $h_{1} \mid W=\mathrm{id}$.

From Theorem 8.2 of [2] we know that $\bar{F}_{1}$, the closure of $F_{1}$ in $I^{\infty}$, has Property $Z$ in $I^{\infty}$. Using Theorem II A of [3] and Theorem 8.5 of [2] there is a homeomorphism $f_{1}$ of $I^{\infty}$ onto itself such that $f_{1}\left(s \cup \bar{F}_{1}\right)=s$. Since $f_{1}\left(W \cap \bar{F}_{1}\right)$ is a compact subset of $s$ we can use Theorem II A of [3] to obtain a homeomorphism $f_{2}$ of $I^{\infty}$ onto itself such that

$$
f_{2}\left[f_{1}\left(W \cap \bar{F}_{1}\right) \cup B\left(I^{\infty}\right)\right]=B\left(I^{\infty}\right) .
$$

Using Lemma 1 we can clearly get a $\beta^{*}$-homeomorphism $f_{3}$ such that $f_{3} \circ f_{2} \circ f_{1} \mid W$ $=$ id. 
Now we note that $f_{3} \circ f_{2} \circ f_{1}\left(\bar{F}_{1} \backslash W\right)=\bigcup_{n=1}^{\infty} C_{n}$, a countable union of compact subsets of $s$. Let $\left\{\gamma_{n}\right\}_{n=1}^{\infty}$ be a collection of pairwise-disjoint, infinite sets of positive integers such that for each $n, \gamma_{n} \subset N \backslash \bigcup_{n=1}^{\infty} \alpha_{i_{n}}$. For each $n$ write $\gamma_{n}=\gamma_{n}^{\prime} \cup \gamma_{n}^{\prime \prime}$ such that $\gamma_{n}^{\prime} \cap \gamma_{n}^{\prime \prime}=\varnothing$ and both are infinite, and where from Theorem 3.5 of [1] we can get a $\beta^{*}$-homeomorphism $f_{4}$ such that $f_{4} \mid W=$ id and $f_{4}\left(C_{n}\right)$ has deficiency with respect to $\alpha_{i_{n}} \cup \gamma_{n}^{\prime}$.

Using Theorem 5.3 of [1] there is a homeomorphism $f_{5}$ of $I^{\infty}$ onto itself such that

$$
f_{5}\left[f_{4} \circ f_{3} \circ f_{2} \circ f_{1}\left(\left(\bar{F}_{1} \cap B\left(I^{\infty}\right)\right) \backslash W\right) \cup B\left(I^{\infty}\right)\right]=B\left(I^{\infty}\right),
$$

$f_{5}$ is the identity on $W$, and $\tau_{i} \circ f_{5}=\tau_{i}$, for all $i \notin \bigcup_{n=1}^{\infty} \gamma_{n}^{\prime}$. Then we put $h_{1}=$ $f_{5} \circ f_{4} \circ f_{3} \circ f_{2} \circ f_{1}$, which is obviously a $\beta^{*}$-homeomorphism satisfying $h_{1} \mid W=$ id. It is also clear that $h_{1}\left(F_{1}\right)=\bigcup_{n=1}^{\infty} A_{n}$, where $A_{n}=f_{5} \circ f_{4}\left(C_{n}\right) \cap s$ is a relatively closed subset of $s$ which is deficient with respect to $\alpha_{i_{n}}$, for all $n$.

We must be able to construct $h_{2}$ arbitrarily close to the identity. Thus let $\varepsilon>0$ be given and let $l$ be a positive integer such that $\left(\sum_{i=l}^{\infty} 2^{2-i}\right)^{1 / 2}<\varepsilon / 6$. Let $\beta_{2}=\left\{j_{1}, j_{2}, \ldots\right\}$ be an infinite, increasing sequence of positive integers such that $\beta_{2} \subset N \backslash \beta_{1}$, $N \mid\left(\beta_{1} \cup \beta_{2}\right)$ is infinite, and $i \in \bigcup_{n=1}^{\infty} \alpha_{j_{n}}$ implies that $i \geqq l$. We will construct a $\beta^{*}$-homeomorphism $h_{2}$ such that $d\left(h_{2}\right.$, id $)<\varepsilon, h_{2} \circ h_{1}\left(F_{2} \mid F_{1}\right)=\bigcup_{n=1}^{\infty} B_{n}$, where each $B_{n}$ is a relatively closed subset of $s$ having deficiency with respect to $\alpha_{j_{n}}$, and $h_{2}$ is the identity on $W_{1} \cup h_{1}\left(W_{1}\right) \cup W \cup h_{1}\left(\bar{F}_{1}\right)$.

From Theorem IIA of [3] there is a homeomorphism $g_{1}$ of $I^{\infty}$ onto itself such that $g_{1}\left(h_{1}\left(\bar{F}_{1} \cup \bar{F}_{2}\right) \cup B\left(I^{\infty}\right)\right)=B\left(I^{\infty}\right)$ and $d\left(g_{1}\right.$, id $)<\varepsilon / 12$. Using Theorem 8.5 of [2] there is a homeomorphism $g_{2}$ of $I^{\infty}$ onto itself such that $g_{2}\left(g_{1} \circ h_{1}\left(\bar{F}_{1} \cup \bar{F}_{2}\right) \cup s\right)$ $=s$. Examining the apparatus used in [2] to obtain $g_{2}$ we find that we may additionally require that $d\left(g_{2}\right.$, id $)<\varepsilon / 12$. Put $A=W_{1} \cup h_{1}\left(W_{1}\right) \cup W$ and note that

$$
g_{2} \circ g_{1}\left[h_{1}\left(\bar{F}_{1} \cap B\left(I^{\infty}\right)\right) \cup\left(A \cap h_{1}\left(\bar{F}_{2}\right)\right)\right]
$$

is a $\sigma$-compact subset of $s$. Thus using Lemma IIA of [3] there is a homeomorphism $g_{3}$ of $I^{\infty}$ onto itself such that

$$
g_{3}\left\{g_{2} \circ g_{1}\left[h_{1}\left(\bar{F}_{1} \cap B\left(I^{\infty}\right)\right) \cup\left(A \cap h_{1}\left(\bar{F}_{2}\right)\right)\right] \cup B\left(I^{\infty}\right)\right\}=B\left(I^{\infty}\right)
$$

and $d\left(g_{3}\right.$, id $)<\varepsilon / 6$. Using Lemma 1 there is a $\beta^{*}$-homeomorphism $g_{4}$ such that $g_{4} \circ g_{3} \circ g_{2} \circ g_{1}$ is the identity on $h_{1}\left(\bar{F}_{1}\right) \cup A$ and $d\left(g_{4}\right.$, id $)<\varepsilon / 3$.

Let $g_{4} \circ g_{3} \circ g_{2} \circ g_{1}\left(h_{1}\left(\bar{F}_{2}\right) \backslash\left(h_{1}\left(\bar{F}_{1}\right) \cup A\right)\right)=\bigcup_{n=1}^{\infty} D_{n}$, a countable union of compact subsets of $s$. Let $\left\{\delta_{n}\right\}_{n=1}^{\infty}$ be a collection of pairwise-disjoint, infinite sets of positive integers such that $\delta_{n} \subset N \backslash \bigcup_{n=1}^{\infty}\left(\alpha_{i_{n}} \cup \alpha_{j_{n}}\right)$ and $m \in \delta_{n}$ implies that $m \geqq l$, for all $n>0$. Then write each $\delta_{n}=\delta_{n}^{\prime} \cup \delta_{n}^{\prime \prime}$ such that $\delta_{n}^{\prime} \cap \delta_{n}^{\prime \prime}=\varnothing$ and both are infinite, and where from Theorem 3.5 of [1] there is a $\beta^{*}$-homeomorphism $g_{5}$ such that $g_{5}\left(D_{n}\right)$ has deficiency with respect to $\alpha_{j_{n}} \cup \delta_{n}^{\prime}$ and $d\left(g_{5}\right.$, id $)<\varepsilon / 6$. If we examine the apparatus used in [1] to obtain $g_{5}$ we find that we may additionally require that $g_{5} \mid\left(h_{1}\left(\bar{F}_{1}\right) \cup A\right)=$ id. 
Now let $g_{6}$ be a homeomorphism of $I^{\infty}$ onto itself such that

$$
\begin{gathered}
g_{6}\left\{\left[g_{5} \circ g_{4} \circ g_{3} \circ g_{2} \circ g_{1}\left(h_{1}\left(\bar{F}_{2}\right) \cap B\left(I^{\infty}\right)\right) \mid\left(h_{1}\left(\bar{F}_{1}\right) \cup A\right)\right] \cup B\left(I^{\infty}\right)\right\}=B\left(I^{\infty}\right), \\
g_{6} \mid\left(h_{1}\left(\bar{F}_{1}\right) \cup A\right)=\mathrm{id}, \quad d\left(g_{6}, \mathrm{id}\right)<\varepsilon / 6,
\end{gathered}
$$

and $\tau_{i} \circ g_{6}=\tau_{i}$, for all $i \notin \bigcup_{n=1}^{\infty} \delta_{n}^{\prime}$. Put $h_{2}=g_{6} \circ g_{5} \circ g_{4} \circ g_{3} \circ g_{2} \circ g_{1}$, which is obviously a $\beta^{*}$-homeomorphism satisfying $h_{2} \mid\left(h_{1}\left(\bar{F}_{1}\right) \cup A\right)=\mathrm{id}$. It is clear that $h_{2} \circ h_{1}\left(F_{2} \mid F_{1}\right)=\bigcup_{n=1}^{\infty} B_{n}$, where $B_{n}=g_{6} \circ g_{5}\left(D_{n}\right) \cap s$ is a relatively closed subset of $s$ which is deficient with respect to $\alpha_{j_{n}}$, for all $n$. This completes the construction of $h_{2}$ and the proof of the lemma.

\section{Separating closed sets with Property $Z$ in $F$-manifolds.}

LEMMA 3. Let $\left\{F_{i}\right\}_{i=1}^{\infty},\left\{K_{j}\right\}_{j=1}^{\infty}$ be collections of relatively closed subsets of $s$, each having Property $Z$ in s, and let $W$ be a finite union of endslices of $I^{\infty}$. Then there exists $a B^{*}$-homeomorphism $h$ such that $h \mid W=\mathrm{id}$ and $h\left(\bigcup_{j=1}^{\infty} K_{j}\right) \cap\left(\bigcup_{i=1}^{\infty} F_{i}\right)=\varnothing$.

Proof. Using Lemma 2 there exists a $\beta^{*}$-homeomorphism $f_{1}$, a collection $\left\{\alpha_{i}\right\}_{i=1}^{\infty}$ of pairwise-disjoint, infinite subsets of $N$, and relatively closed subsets $\left\{C_{i}\right\}_{i=1}^{\infty}$ of $s$, each $C_{i}$ having deficiency with respect to $\alpha_{i}$, such that $f_{1} \mid W=$ id and $f_{1}\left(\bigcup_{i=1}^{\infty} F_{i}\right)=\bigcup_{i=1}^{\infty} C_{i}$.

Applying Lemma 2 to $\left\{K_{j}\right\}_{j=1}^{\infty}$ we obtain a $\beta^{*}$-homeomorphism $f_{2}$, a collection $\left\{\beta_{j}\right\}_{j=1}^{\infty}$ of pairwise-disjoint, infinite sets of positive integers such that each $\beta_{j}$ is constructed by choosing exactly one element from each $\alpha_{i}$, and a collection $\left\{D_{j}\right\}_{j=1}^{\infty}$ of relatively closed subsets of $s$ such that each $D_{j}$ is deficient with respect to $\beta_{j}, f_{2} \mid W=\mathrm{id}$, and $f_{2}\left(\bigcup_{j=1}^{\infty} K_{j}\right)=\bigcup_{j=1}^{\infty} D_{j}$.

We will now construct a $\beta^{*}$-homeomorphism $g$ such that $g \mid W=$ id and $g\left(D_{j}\right)$ $\cap C_{i}=\varnothing$, for all $i$ and $j$. For each $i$ and $j$ let $g_{i, j}$ be a $\beta^{*}$-homeomorphism which satisfies the following conditions

(1) $\tau_{k} \circ g_{i, j}=\tau_{k}$, for $k \notin \alpha_{i} \cap \beta_{j}$,

(2) $\tau_{\alpha_{i} \cap \beta_{j}} \circ g_{i, j}\left(D_{j}\right) \neq \tau_{\alpha_{i} \cap \beta_{j}}\left(C_{i}\right)$,

(3) $g_{i, j} \mid W=\mathrm{id}$, and

(4) $g_{i, j}\left(W_{n}^{+}\right)=W_{n}^{+}$and $g_{i, j}\left(W_{n}^{-}\right)=W_{n}^{-}$, for all $n>0$.

It is clear that (3) can be achieved by phasing out the motion isotopically as we approach $W$. Then we define

$$
g=L \prod_{n=1}^{\infty}\left(g_{1, n} \circ g_{2, n-1} \circ \cdots \circ g_{n, 1}\right)
$$

which satisfies our requirements. It is clear that $h=f_{1}^{-1} \circ g \circ f_{2}$ satisfies the conditions of the lemma.

Lemma 4. Let $\left\{F_{i}\right\}_{i=1}^{\infty}$ and $\left\{K_{j}\right\}_{j=1}^{\infty}$ be collections of closed subsets of any $F$ manifold $X$, with each $F_{i}$ and $K_{j}$ having Property $Z$ in $X$. Then there is a homeomorphism $h$ of $X$ onto itself such that $h\left(\bigcup_{j=1}^{\infty} K_{j}\right) \cap\left(\bigcup_{i=1}^{\infty} F_{i}\right)=\varnothing$. 
Proof. Using Henderson's open embedding theorem let $f: X \rightarrow s$ be an embedding of $X$ as an open subset of $s$. From Theorem 1 of [5] there is a star-finite open cover $\left\{U_{k}\right\}_{k=1}^{\infty}$ of $f(X)$, where each $U_{k}$ is a basic open subset of $s$ contained in $f(X)$. Moreover let us assume that the $U_{k}$ 's are indexed as in Convergence Procedure B.

Note that for each $k>0, \bar{U}_{k}$ is a Hilbert cube, where $\bar{U}_{k}$ is the closure of $U_{k}$ in $I^{\infty}$, and $U_{k}$ may be regarded as the relative pseudo-interior of $\bar{U}_{k}$. It is obvious that the topological boundary of $U_{k}$ in $s$ is contained in a finite union of endslices of the relative pseudo-boundary of $\bar{U}_{k}$. We can then apply Lemma 3 to obtain a homeomorphism $g_{1}$ of $f(X)$ onto itself such that $g_{1}|f(X)| U_{1}=$ id and

$$
g_{1}\left(\bigcup_{j=1}^{\infty} f\left(K_{j}\right)\right) \cap\left(\bigcup_{i=1}^{\infty} f\left(F_{i}\right)\right) \cap U_{1}=\varnothing .
$$

Suppose now that homeomorphisms $\left\{g_{k}\right\}_{k=1}^{n}$ of $f(X)$ onto itself have been defined such that $g_{k}|f(X)| U_{k}=$ id and

$$
\left(g_{k} \circ \cdots \circ g_{1}\right)\left(\bigcup_{j=1}^{\infty} f\left(K_{j}\right)\right) \cap\left(\bigcup_{i=1}^{\infty} f\left(F_{i}\right)\right) \cap U_{k}=\varnothing,
$$

for $1 \leqq k \leqq n$. Once more apply Lemma 3 to obtain a homeomorphism $g_{n+1}$ of $f(X)$ onto itself such that $g_{n+1}|f(X)| U_{n+1}=$ id and

$$
\left(g_{n+1} \circ \cdots \circ g_{1}\right)\left(\bigcup_{j=1}^{\infty} f\left(K_{j}\right)\right) \cap\left(\bigcup_{i=1}^{\infty} f\left(F_{i}\right)\right) \cap U_{n+1}=\varnothing .
$$

It is clear that $g=L \prod_{k=1}^{\infty} g_{k}$ defines a homeomorphism of $f(X)$ onto itself such that $g\left(\bigcup_{j=1}^{\infty} f\left(K_{j}\right)\right) \cap\left(\bigcup_{i=1}^{\infty} f\left(F_{i}\right)\right)=\varnothing$. Then $h=f^{-1} \circ g \circ f$ satisfies the requirements of our lemma.

5. Proof of Theorem 1. We break Theorem 1 up into two lemmas, the first treating the case $T=I^{\infty}$ and the second treating the case $T=s$.

LEMMA 5. Let $X$ be any F-manifold and let $\left\{K_{i}\right\}_{i=1}^{\infty}$ be a collection of closed subsets of $X \times I^{\infty}$, with each $K_{i}$ having Property $Z$ in $X \times I^{\infty}$. Then there is a homeomorphism $h$ of $X \times I^{\infty}$ onto itself and a collection $\left\{\alpha_{i}\right\}_{i=1}^{\infty}$, where the $\alpha_{i}$ 's are pairwise-disjoint, infinite sets of positive integers, such that for all $i>0, h\left(K_{i}\right)$ is deficient with respect to $\alpha_{i}$ in $X \times I^{\infty}$.

Proof. For each positive integer $j$ let

$$
F_{j}^{+}=\left\{(x, y) \in X \times I^{\infty} \mid \tau_{j}(y)=1\right\}, \quad F_{j}^{-}=\left\{(x, y) \in X \times I^{\infty} \mid \tau_{j}(y)=-1\right\},
$$

and $F_{j}=F_{j}^{+} \cup F_{j}^{-}$. A straightforward and standard argument shows that each $F_{j}$ has Property $Z$ in $X \times I^{\infty}$. Thus applying Lemma 4 to $X \times I^{\infty}$ there is a homeomorphism $f_{1}$ of $X \times I^{\infty}$ onto itself such that $f_{1}\left(\bigcup_{i=1}^{\infty} K_{i}\right) \cap\left(\bigcup_{j=1}^{\infty} F_{j}\right)=\varnothing$.

Let $j$ and $m$ be any positive integers and for each $x \in X \times \prod_{i \neq j} I_{i}$ let $U_{x} \times\left(\delta_{x}, 1\right]$ 
be an open subset of $\left(X \times \prod_{i \neq j} I_{i}\right) \times I_{j}$ containing $(x, 1)$ such that $\left(U_{x} \times\left(\delta_{x}, 1\right]\right)$ $\cap f_{1}\left(K_{m}\right)=\varnothing$. Note that $\left\{U_{x} \mid x \in X \times \prod_{i \neq j} I_{i}\right\}$ is an open cover of $X \times \prod_{i \neq j} I_{i}$. Since we have already noted that $X \times \prod_{i \neq j} I_{i}$ is homeomorphic to $X$, and is hence an $F$-manifold, we can apply Lemma 5 of [5] to get a star-finite open refinement $\left\{U_{i}\right\}_{i=1}^{\infty}$ of $\left\{U_{x} \mid x \in X \times \prod_{i \neq j} I_{i}\right\}$ which covers $X \times \prod_{i \neq j} I_{i}$. Moreover we may assume that the $U_{i}$ 's are indexed as in Convergence Procedure B. It is clear that for each $i>0$ there is a number $\delta_{i}$ such that $1 / 2 \leqq \delta_{i} \leqq 1$ and $\left(U_{i} \times\left(\delta_{i}, 1\right]\right) \cap f_{1}\left(K_{m}\right)=\varnothing$.

Now construct a closed cover $\left\{C_{i}\right\}_{i=1}^{\infty}$ of $X \times \prod_{i \neq i} I_{i}$ such that $C_{i} \subset U_{i}$, for all $i>0$. For each $i>0$ let $\varphi_{i}: X \times \prod_{i \neq j} I_{i} \rightarrow[0,1]$ be a continuous function such that $\varphi_{i}(x)=1$ for $x \in C_{i}$ and $\varphi_{i}(x)=0$ for $x \in\left(X \times \prod_{i \neq j} I_{i}\right) \backslash U_{i}$. For each $i>0$ we now construct a homeomorphism $g_{i}$ of $\left(X \times \prod_{i \neq j} I_{i}\right) \times I_{j}$ onto itself which slides points linearly in the $I_{j}$-direction as follows: if $x \in X \times \prod_{i \neq j} I_{i}$, then $\{x\} \times I_{j}$ is taken linearly onto itself such that $g_{i}\left(x, \delta_{i}\right)=\left(x, \delta_{i}\left(1-\varphi_{i}(x)\right)+(1 / 2) \varphi_{i}(x)\right)$. Applying Convergence Procedure $\mathrm{B}^{\prime}$ to $\left\{g_{i}\right\}_{i=1}^{\infty}$ let $g=L \prod_{i=1}^{\infty} g_{i}$, which is a homeomorphism of $X \times I^{\infty}$ onto itself satisfying $g \circ f_{1}\left(K_{m}\right) \subset X \times \prod_{i \neq j} I_{i} \times[-1,1 / 2]$. Using similar techniques we can construct a homeomorphism $g^{\prime}$ of $X \times I^{\infty}$ onto itself which slides points only in the $I_{j}$-direction such that $g^{\prime} \circ g \circ f_{1}\left(K_{m}\right) \subset X \times \prod_{i \neq j} I_{i} \times[-1 / 2,1 / 2]$.

Choose a collection $\left\{\beta_{i}\right\}_{i=1}^{\infty}$ of pairwise-disjoint, infinite sets of integers such that $N=\bigcup_{i=1}^{\infty} \beta_{i}$. Then we can successively use the above construction to obtain a homeomorphism $f_{2}$ of $X \times I^{\infty}$ onto itself such that $\tau_{j} \circ \pi_{l^{\infty}} \circ f_{2} \circ f_{1}\left(K_{i}\right) \subset[-1 / 2,1 / 2]$, for all $j \in \beta_{i}$ and all $i>0$.

Using Theorem 3.5 of [1] there is a homeomorphism $d_{i}$ of $I^{\beta_{i}}$ onto itself such that $d_{i}\left(\prod_{j \in \beta_{i}}[-1 / 2,1 / 2]_{j}\right)$ has deficiency with respect to an infinite subset $\alpha_{i}$ of $\beta_{i}$, for all $i>0$. Grouping these together we obtain a homeomorphism $d$ of $X \times I^{\infty}$ $=X \times I^{\beta_{1}} \times I^{\beta_{2}} \times \cdots$ onto itself defined by $d=\left(\mathrm{id}, d_{1}, d_{2}, \ldots\right)$. Then $h=d \circ f_{2} \circ f_{1}$ is a homeomorphism of $X \times I^{\infty}$ onto itself such that $h\left(K_{i}\right)$ is deficient with respect to $\alpha_{i}$, for all $i>0$.

LEMMA 6. Let $X$ be any F-manifold and let $\left\{K_{i}\right\}_{i=1}^{\infty}$ be a collection of closed subsets of $X \times s$, with each $K_{i}$ having Property $Z$ in $X \times s$. Then there is a homeomorphism $h$ of $X \times s$ onto itself and a collection $\left\{\alpha_{i}\right\}_{i=1}^{\infty}$, where the $\alpha_{i}$ 's are pairwise-disjoint, infinite sets of positive integers, such that for all $i>0, h\left(K_{i}\right)$ is deficient with respect to $\alpha_{i}$ in $X \times s$.

Proof. Using Theorem 9.3 of [1] there is a homeomorphism $f$ of $s=\prod_{i=1}^{\infty} I_{i}^{0}$ onto $s^{\alpha} \times I^{\beta}$, where $\alpha$ is the set of even positive integers and $\beta$ is the set of odd positive integers. Let $\bar{f}$ be the homeomorphism of $X \times s$ onto $X \times s^{\alpha} \times I^{\beta}$ defined by $\bar{f}(x, y)=(x, f(y))$, where $(x, y) \in X \times s$.

From Lemma 5 there is a homeomorphism $g$ of $\left(X \times s^{\alpha}\right) \times I^{\beta}$ onto itself such that $\pi_{I^{\beta}} \circ g \circ \bar{f}\left(K_{i}\right)$ is deficient with respect to $\beta_{i}$, where the $\beta_{i}$ 's are pairwise-disjoint, infinite, and $\beta=\bigcup_{i=1}^{\infty} \beta_{i}$. Now write $\alpha=\bigcup_{i=1}^{\infty} \alpha_{i}^{\prime}$, where the $\alpha_{i}^{\prime}$ 's are pairwise-disjoint and infinite. Furthermore write $\alpha_{i}^{\prime}=\bigcup_{j=1}^{\infty} \alpha_{i, j}$, where for each $i>0$ the $\alpha_{i, j}$ 's are 
pairwise-disjoint and infinite, and write $\beta_{i}=\left\{m_{i, 1}, m_{i, 2}, \ldots\right\}$, an increasing sequence of integers.

We note that for each $i$ and $j$ we have

$$
\tau_{k} \circ \pi_{s^{\alpha} \times I^{\beta}} \circ g \circ \bar{f}\left(K_{i}\right) \subset s^{\alpha, j} \times\left\{a_{i, j}\right\},
$$

where $a_{i, j} \in I_{m_{i, j}}^{0}$ and $k=\alpha_{i, j} \cup\left\{m_{i, j}\right\}$. Theorem 9.3 of [1] gives a homeomorphism of $\prod_{i=1}^{\infty} I_{i}^{0} \times[-1,1]$ onto $\prod_{i=1}^{\infty} I_{i}^{0} \times(-1,1)$. If we examine the apparatus used to prove this we find that for each $i$ and $j$ we can get a homeomorphism $d_{i, j}$ of $s^{\alpha_{i, j}} \times I_{m_{i, j}}$ onto $s^{\alpha_{i, j}} \times I_{m_{i, j}}^{0}$ such that $d_{i, j}\left(s^{\alpha_{i, j}} \times\left\{a_{i, j}\right\}\right)=s^{\alpha_{i, j}} \times\left\{a_{i, j}\right\}$. This then gives a homeomorphism $d$ of $X \times s^{\alpha} \times I^{\beta}$ onto $X \times s^{\alpha} \times s^{\beta}$ such that $d \circ g \circ \bar{f}\left(K_{i}\right)$ is deficient with respect to $\beta_{i}$, for all $i>0$. Thus with $h=d \circ g \circ \bar{f}$ and $\left\{\alpha_{i}\right\}_{i=1}^{\infty}=\left\{\beta_{i}\right\}_{i=1}^{\infty}$ the proof of the lemma is complete.

6. Proof of Theorem 2. Using Theorem 1 there is a homeomorphism $f_{1}$ of $X$ onto $X \times s$ and a collection $\left\{\alpha_{i}\right\}_{i=1}^{\infty}$ of pairwise-disjoint, infinite sets of positive integers such that for each $i>0, f_{1}\left(K_{i}\right)$ is deficient with respect to $\alpha_{i}$ in $X \times s$.

Applying Henderson's open embedding theorem let $f_{2}: X \rightarrow s_{1}$ be an open embedding, where $s_{1}=\prod\left\{I_{i}^{0} \mid i\right.$ odd $\}$. If $s_{2}=\prod\left\{I_{i}^{0} \mid i\right.$ even $\}$ then there is a homeomorphism $f_{3}$ of $s$ onto $s_{2}$ which is defined coordinatewise. Thus $f_{4}=f_{2} \times f_{3}$ is an open embedding of $X \times s$ into $s=s_{1} \times s_{2}$ and there is a collection $\left\{\beta_{i}\right\}_{i=1}^{\infty}$ of pairwisedisjoint, infinite sets of positive even integers such that $f_{4} \circ f_{1}\left(K_{i}\right)$ is deficient with respect to $\beta_{i}$, for all $i>0$.

If $\mathrm{Cl}\left(f_{4} \circ f_{1}\left(K_{i}\right)\right)$ is the closure of $f_{4} \circ f_{1}\left(K_{i}\right)$ in $I^{\infty}$, then $\mathrm{Cl}\left(f_{4} \circ f_{1}\left(K_{i}\right)\right) \backslash f_{4} \circ f_{1}\left(K_{i}\right)$ $=\bigcup_{j=1}^{\infty} C_{i, j}$, a countable union of compact subsets of $I^{\infty}$, with each $C_{i, j}$ having deficiency with respect to $\beta_{i}$, for all $i>0$. Using Theorem 5.3 of [1] there is a homeomorphism $g$ of $I^{\infty}$ onto itself such that $g\left[\left(\bigcup_{i=1}^{\infty} \bigcup_{j=1}^{\infty} C_{i, j}\right) \cup B\left(I^{\infty}\right)\right]=B\left(I^{\infty}\right)$ $\tau_{i} \circ g=\tau_{i}$, for $i$ odd, and $g \circ f_{4} \circ f_{1}\left(K_{i}\right)$ has infinite deficiency, for all $i>0$. Clearly $h=g \circ f_{4} \circ f_{1}$ is the required embedding.

\section{REFERENCES}

1. R. D. Anderson, Topological properties of the Hilbert cube and the infinite product of open intervals, Trans. Amer. Math. Soc. 126 (1967), 200-216. MR 34 \#5045.

2. - On topological infinite deficiency, Michigan Math. J. 14 (1967), 365-383. MR 35 \#4893.

3. - Strongly negligible sets in Fréchet manifolds, Bull. Amer. Math. Soc. 75 (1969), 64-67.

4. R. D. Anderson and R. Schori, Factors of infinite-dimensional manifolds, Trans. Amer. Math. Soc. 142 (1969), 315-330.

5. R. D. Anderson, David W. Henderson and James E. West, Negligible subsets of infinitedimensional manifolds, Compositio Math. 21 (1969), 143-150.

6. William Barit, Notices Amer. Math. Soc. 16 (1969), 295, Abstract \#663-715.

7. C. Bessaga and A. Pełczyński, Estimated extension theorem, homogeneous collections 
and skeletons, and their applications to topological classifications of linear metric spaces and convex sets, Fund. Math. (to appear).

8. David W. Henderson, Infinite-dimensional manifolds are open subsets of Hilbert space, Bull. Amer. Math. Soc. 75 (1969), 759-762.

9. H. Torunczyk, Remarks on Anderson's paper on topological infinite deficiency, Fund. Math. (to appear)

\section{Louisiana State University, \\ BAton Rouge, Louisiana}

